\title{
CSA-dependent degradation of CSB by the ubiquitin- proteasome pathway establishes a link between complementation factors of the Cockayne syndrome
}

\begin{abstract}
Regina Groisman, ${ }^{1,3,8}$ Isao Kuraoka, ${ }^{5}$
Odile Chevallier, ${ }^{6}$ Nogaye Gaye, ${ }^{3}$

Thierry Magnaldo, ${ }^{6}$ Kiyoji Tanaka, ${ }^{5}$ Alexei F. Kisselev, ${ }^{2,4,7}$ Annick Harel-Bellan, ${ }^{3,7}$ and Yoshihiro Nakatani ${ }^{1,7}$

${ }^{1}$ Dana-Farber Cancer Institute, ${ }^{2}$ Department of Cell Biology, Harvard Medical School, Boston, Massachusetts 02115, USA; ${ }^{3}$ UPR9079 CNRS-Ligue Nationale Contre le Cancer, 94800 Villejuif, France; ${ }^{4}$ Norris Cotton Cancer Center and Department of Pharmacology and Toxicology, Dartmouth Medical School, Lebanon, New Hampshire 03756, USA; ${ }^{5}$ Graduate School of Frontier Biosciences, Osaka University, and Core Research for Evolutional Science and Technology, Japan Science and Technology Agency, Suita, Osaka 565-0871, Japan; ${ }^{6}$ Laboratory of Genetic Instability and Cancer, CNRS UPR2169, Institut Gustave Roussy, Villejuif 94805, France
\end{abstract}

Mutations in the CSA or CSB complementation genes cause the Cockayne syndrome, a severe genetic disorder that results in patients' death in early adulthood. CSA and CSB act in a transcription-coupled repair (TCR) pathway, but their functional relationship is not understood. We have previously shown that CSA is a subunit of an E3 ubiquitin ligase complex. Here we demonstrate that CSB is a substrate of this ligase: Following UV irradiation, CSB is degraded at a late stage of the repair process in a proteasome- and CSA-dependent manner. Moreover, we demonstrate the importance of CSB degradation for post-TCR recovery of transcription and for the Cockayne syndrome. Our results unravel for the first time the functional relationship between CSA and CSB.

Received December 30, 2005; revised version accepted March $27,2006$.

DNA damage represents a major threat for the maintenance of genomic integrity, and a variety of cellular pathways recognize and repair defects in DNA structure. Repair of UV-light-induced pyrimidine dimers or of adducts created by cisplatin is carried out by the nucleotide excision repair (NER) pathway. This pathway is impaired in several diseases such as Xeroderma pigmentosum $(\mathrm{XP})$, Cockayne syndrome (CS), trichothiodystrophy

[Keywords: CSB; CSA; ubiquitin-proteasomal degradation]

${ }^{7}$ These authors contributed equally to this work.

${ }^{8}$ Corresponding author.

E-MAIL groisman@vjf.cnrs.fr; FAX 33-1-49583307.

Article is online at http://www.genesdev.org/cgi/doi/10.1101/gad.378206.
(TTD), and the mild ultraviolet (UV)-light-sensitive syndrome (Bootsma et al. 1998).

NER proceeds via two alternative pathways: The global genome repair (GGR) is involved in the repair of any sequence in the genome regardless of its transcriptional status; the transcription-coupled repair (TCR) is only involved in the repair of actively transcribed DNA strands. TCR occurs at a higher rate than GGR, but the reason for this difference is not fully understood. Most of the events of the two pathways are identical; in both cases, DNA unwinding is followed by excision of a 27-30nucleotide oligonucleotide fragment containing the photoproduct of the damaged DNA strand and its replacement by de novo synthesis using the opposite, untouched, DNA strand as the template. Thus, the major difference between GGR and TCR occurs at the level of recognition of the DNA damage. In the GGR pathway, the damage is initially recognized via a direct interaction of NER proteins XPE and XPC-HR23B with damaged DNA. In contrast, in TCR, damages appear to be signaled via the stalling of RNA polymerase II ( $\mathrm{Pol}$ II). The release of RNA polymerase involves two proteins, CSA and CSB, but their mode of action is unknown (Friedberg et al. 1995; Svejstrup 2002).

CSA and CSB are TCR factors, the mutation of which causes the Cockayne syndrome. CSB is a member of the SWI2/SNF2 family of ATP-dependent chromatin remodeling factors and has the activities of SWI2/SNF2 proteins (Troelstra et al. 1992): DNA-dependent ATPase (but not classical helicase) (Selby and Sancar 1997b; Citterio et al. 1998), nucleosome remodeling, and interaction with core histones (Citterio et al. 2000). In addition, CSB locally influences the DNA conformation, likely by wrapping the DNA around itself (Beerens et al. 2005), thereby modifying the interface between stalled RNA polymerase II and DNA. This modification promotes DNA repair or allows the bypass of damage (Svejstrup 2003).

The role of CSB in general transcription is a controversial issue. Several studies proposed that CSB is promoting elongation by RNA polymerase I (Bradsher et al. 2002), II (Selby and Sancar 1997a; Tantin et al. 1997), and III (Yu et al. 2000). However, another study does not support this model: CSB counteracted the rescue of backtracked and arrested transcription complexes by the elongation factor TFIIS (Selby and Sancar 1997b). Moreover, CSB knockout mice, as well as some patients lacking CSB, display much milder growth-related and neurological defects than Cockayne syndrome patients with mutant CSB, which does not support a requirement for CSB in general transcription (van der Horst et al. 1997; Horibata et al. 2004).

The CSA gene encodes a protein of $46 \mathrm{kDa}$ with five WD-40 repeats that associates with cullin 4A (CUL4A) containing E3 ubiquitin ligase (Groisman et al. 2003). CSA physically associates with RNA Pol II in a UV-dependent manner (Kamiuchi et al. 2002; Groisman et al. 2003).

In our previous work, we have demonstrated that the TCR-associated factor CSA and the GGR-associated factor DDB2 form very similar E3 ubiquitin-ligase complexes, establishing a link between these two pathways and ubiquitin-dependent protein degradation (Groisman 
et al. 2003). The E3 ligase activity of both complexes was down-regulated in response to UV irradiation by the COP9/signalosome (CSN), a protein complex that associates with ubiquitin isopeptidase activity (Groisman et al. 2003; Wolf et al. 2003). However, the kinetics of activation of these ligases was completely different. The DDB2 ligase was active immediately after UV irradiation, and inhibited by association with CSN at later times. In contrast, CSA ligase was silenced by CSN at the beginning of the repair process, and became active at later stages (Groisman et al. 2003). We suggested that the CSA-associated ligase is responsible for the degradation of TCR repair factors at the end of the repair process, a degradation process that would be needed for transcription to resume. Consistent with this hypothesis, it was previously shown that proteasome inhibitors specifically interfere with the efficiency of mRNA synthesis recovery, but not with the repair process (McKay et al. 2001).

In the present study, we have tested the hypothesis that CSB itself could be a substrate for CSA: We demonstrate that CSB is ubiquitinylated by the CSA ligase and degraded by the proteasome in a UV-dependent manner at a late stage of the repair process, and that CSB degradation impacts on the recovery of RNA synthesis after TCR.

\section{Results and Discussion}

In vitro, a physical interaction between CSA and CSB was reported by Henning et al. (1995). However, no association has been found in cells using gel filtration, coimmunoprecipitation, or immunofluorescence (Tantin et al. 1997; van Gool et al. 1997; Bradsher et al. 2002). Therefore, it is generally thought that in cells, interactions between CSA and CSB are weak and perhaps transient (Licht et al. 2003), as usually observed for interactions between enzymes and their substrates.

In order to test whether CSB interacts with CSA, we immunoprecipitated CSA from HeLa cells expressing a Flag/HA-tagged version of CSA (eCSA/HeLa) with antiFlag antibodies. CSB was coprecipitated with CSA (Fig. 1A, lanes 1,2). Similar experiments with HeLa cells expressing the epitope-tagged DDB2 showed that there is no interaction between DDB2 and CSB (Fig. 1A, lanes $3,4)$, although a weak interaction can be seen under certain experimental conditions (data not shown). Thus, CSB interacts specifically with CSA.

This observation of the CSA-CSB interaction is in contradiction with the results of van Gool et al. (1997), who did not detect CSB with endogenous CSA following size fractionation. In order to explore the reason for this discrepancy, we similarly analyzed our complex on a glycerol gradient (Fig. 1B). Under our conditions, CSA fractionated into two peaks: a peak containing CSA in association with COP9/CSN in fractions 14-19, and a peak devoid of CSN/COP9 in fractions 10-12. Notably, CSB was found associated with CSA only in the presence of COP9/CSN (fractions 14-19), suggesting that COP9/ CSN stabilizes CSB-CSA interactions. Thus, a likely hypothesis is that our separation procedure detects two distinct complexes, whereas the procedure used by van Gool et al. detects only one complex: the CSA complex devoid of COP9/CSN.

The physical association between CSA and CSB raises the possibility that CSB is a substrate for CSA. The E3 ligase activity associated with CSA is up-regulated for
A

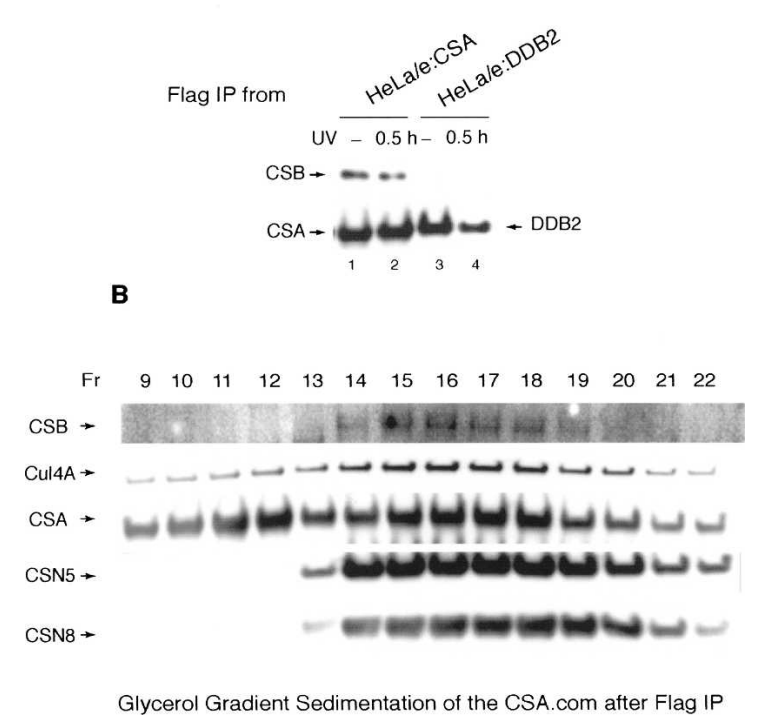

Figure 1. Characterization of the interaction between CSA and CSB. (A) Immunoblot detection of CSB protein in Flag-CSA (lanes 1,2) and Flag-DDB2 (lanes 3,4) complexes immunoprecipitated (IP) with anti-Flag antibodies from HeLa cell extract. $(B)$ Glycerol gradient sedimentation analysis of the Flag-CSA complex. Immunoblot analysis of the distribution of CSB, CSN, and Cul4A in fractions of the gradient.

several hours following UV irradiation (Groisman et al. 2003). Thus, we tested whether CSA ubiquitin ligase activity up-regulation was concomitant with CSB ubiquitination and subsequent degradation by the proteasome. A time-course analysis of the complex showed that CSB was associated with CSA during the early steps of UV response, but disappeared $3 \mathrm{~h}$ after UV irradiation (Fig. $2 \mathrm{~A}$, lanes 7,91 , at a time corresponding to activation of the ligase (Groisman et al. 2003). Moreover, in the presence of the proteasome inhibitor MG132, CSB could be detected in association with CSA at all times (Fig. 2A, lanes 8,10$)$, suggesting that its disappearance after UV irradiation is a consequence of proteasomal degradation. Thus, CSB binds CSA at early stages of DNA repair and is subsequently removed from the CSA complex by proteasome-dependent degradation at later stages.

To provide further evidence that CSB degradation is CSA-dependent, we measured CSB levels in immortalized CSA-deficient fibroblasts that were derived from Cockayne syndrome patients (Bregman et al. 1996). In normal fibroblasts, as in HeLa cells, CSB levels decreased dramatically $4 \mathrm{~h}$ after UV irradiation (Fig. 2B, cf. lanes 1 and 3). Cell treatment with MG-132 prevented CSB disappearance (Fig. 2B, lane 4), directly demonstrating that CSB is also degraded by the proteasome in normal fibroblasts. In contrast, in CSA-deficient fibroblasts, CSB levels remained stable after UV irradiation, and were not modified by cell treatment with MG132 (Fig. 2, lanes 5-8). Moreover, in rescue experiments, CSA-deficient fibroblasts stably transfected with exogenous myc-tagged CSA recovered their ability to degrade CSB $4 \mathrm{~h}$ after UV irradiation (Fig. 2B, lanes 9-12). Thus, CSA not only interacts with CSB but also is required for UV-induced degradation of CSB. Our data, however, are in contradiction with the results of Rockx et al. (2000), who also 
A

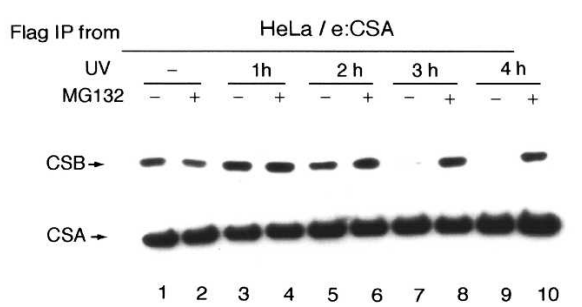

B

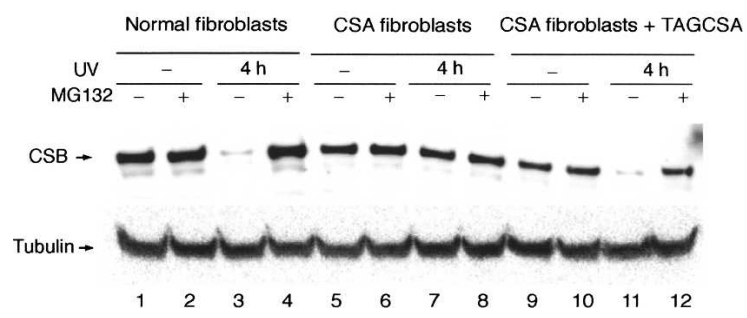

Figure 2. UV-induced proteasomal degradation of CSB depends on CSA. (A) Association of CSB with Flag-CSA is stabilized by MG132. Detection of CSB on immunoblot of the Flag-CSA complexes purified from control (lanes 1,2) and UV-irradiated (lanes 3,4) eCSA: HeLa cells harvested at $1 \mathrm{~h}$ (lanes 3,4), $2 \mathrm{~h}$ (lanes 5,6), $3 \mathrm{~h}$ (lanes 7,8), and $4 \mathrm{~h}($ lanes 9,10$)$ after UV irradiation. Where indicated, proteasome inhibitor MG132 was added $1 \mathrm{~h}$ prior to UV-irradiation. (B) Endogenous CSB degradation occurs in normal fibroblasts $4 \mathrm{~h}$ after UV irradiation (lanes 1-4) and is disturbed in the CSA fibroblasts (lanes 5-8), but is restored after transfection of exogenous myc-CSA (lanes 9-12). CSB levels in the whole-cell lysates were measured on immunoblots with anti-CSB antibodies. Note that samples and controls were run simultaneously on independent gels.

found down-regulation of CSB during repair but independent of CSA expression. These authors used cell lines established by SV40 transformation. It is likely that alternative pathways that are not normally used have been selected during the long-term establishment of SV40transformed cell lines.

In order to directly demonstrate that CSA is responsible for CSB ubiquitinylation, we used an in vitro assay. Purified recombinant CSB was incubated with ATP, ubiquitin, E1, E2 (UbcH5), and the CSA ligase complex, which was reconstituted from components overexpressed in insect cells (Fig. 3A). A high-molecular-weight smear characteristic of a polyubiquitinated protein was detected with CSB antibodies. Moreover, the amount of ubiquitinated CSB was proportional to the amount of CSA E3 ligase added to the reaction, suggesting that ubiquitination of CSB is dependent on the CSA-DDB1CUL4A-Roc1 complex (Fig. 3B, lanes 5-8). Omission of E1, E2, or ubiquitin abolished formation of the reaction product, confirming that it was indeed ubiquitinated CSB (Fig. 3B, lanes 1-4). Notably, the DDB2 E3 ligase complex, of highly similar composition and differing only by the replacement of CSA by DDB2 (Fig. 3C), does not polyubiquitinate CSB (Fig. 3D) although the ligase is active, judging from auto-ubiquitination of CUL4A in the complex (Fig. 3D, bottom panel; note that a slight shift in CSB migration was observed after incubation with the DDB2 ligase, which may correspond to monoubiquitinated CSB). These results demonstrate that DDB2 cannot replace CSA in the CSB polyubiquitination reaction. Moreover, they imply that CSA, and not any other component of the complex such as DDB1 that is common to both complexes, is the substrate recognition subunit of the ligase.

All cullin-RING ubiquitin ligases consist of at least four components (Petroski and Deshaies 2005): a cullin scaffold, a RING finger, a substrate receptor (an F-box or a SOCS-box protein, or a BTB-domain protein), and an adaptor protein (Skp1, elongin BC, or BTB-domain protein) that connects the receptor to the cullin. We next tested the hypothesis that DDB1 functions as an adaptor connecting substrate receptors CSA and DDB2 (WD40 proteins) to cullin 4A. We coinfected insect Sf9 cells with baculoviruses expressing GST-Cul4A, Roc1, HADDB1, and Flag-DDB2 or Flag-CSA in different combinations and performed GST pull-down assays. Only when DDB1 was expressed, was GST-Cul4A able to efficiently pull down Flag-CSA or Flag-DDB2 (Fig. 4, lanes 3,8 ). In the absence of DDB1, neither CSA nor DDB2

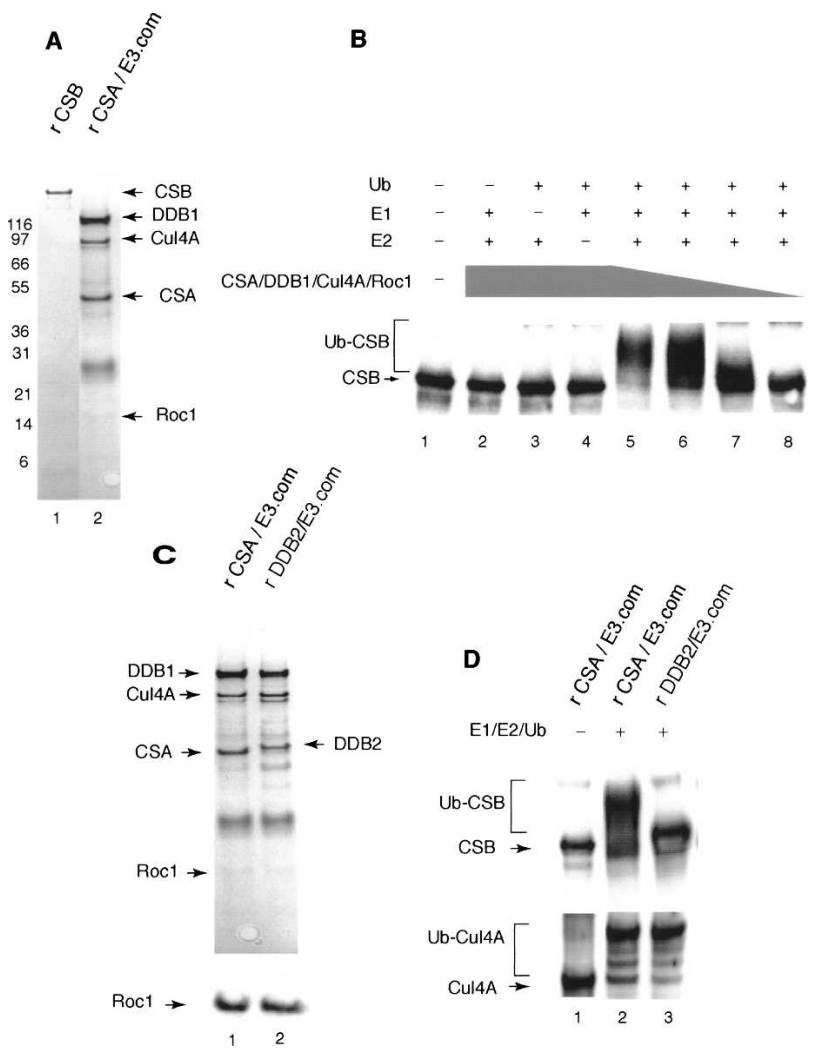

Figure 3. CSB ubiquitination by CSA ligase in vitro. (A) SDS-PAGE of the purified CSB (lane 1) and CSA ligase complex reconstituted from purified GST-CUL4A, HA-DDB1, Flag-CSA, and Roc1 expressed by baculovirus in insect cells (lane 2). The gel was stained with Coomassie. The presence of Roc1 in the CSA ligase complex was confirmed by immunoblot (as shown in lane 1 in $C$ ). $(B)$ Western blot analysis of the ubiquitination reaction. Different amounts of reconstituted CSA ligase were incubated with recombinant CSB for $1 \mathrm{~h}$ in the presence (lanes 5-8) or absence (lanes 1-4) of other components of the ubiquitination reaction as indicated. Ubiquitinated CSB was detected with anti-CSB antibodies. $(C)$ Amount and purity of DDB2 ligase (lane 2) as compared with the CSA ligase (lane 1) by the Coomassie-stained gel. Roc1 was detected by immunoblotting. $(D)$ Comparison of the ubiquitin-ligase activity of DDB2 (lane 3) and CSA (lane 2) complexes using CSB as a substrate (top panel) and in a CUL4A auto-ubiquitination reaction (bottom panel). Immunoblots were developed with anti-CSB (top panel) or anti-cul4A (bottom panel) antibodies. 


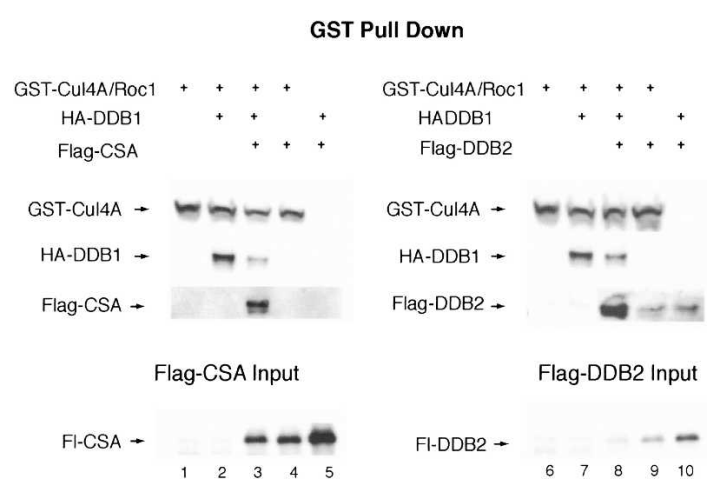

Figure 4. DDB1 is an adaptor protein for cullin 4A-containing ligases. GST-pull-down experiment of E3 ligase components coexpressed by baculovirus with GST-CUL4A. Note that a significant amount of CSA (lane 3) and DDB2 (lane 8) was detected by IP only if coexpressed with DDB1. In the absence of DDB1 expression, CSA did not bind CUL4A (lane 4) and the amount of DDB2 in the complex was reduced dramatically (lane 9). The bottom panel represents $20 \%$ input for CSA (lanes 1-5) and DDB2 (lanes 6-10).

interacted with cul4A (Fig. 4, lanes 4,9). These results strongly suggest that DDB1 is an adaptor for CSA and DDB2 ligases, and is therefore required for the degradation of CSB and other substrates of these ligases. The conclusion that WD-40 proteins serve as substrate receptors in the cullin 4A-containing ubiquitin ligases is further strengthened by the recent study demonstrating that another WD-40 repeat protein, Cdt2 (Liu et al. 2005), serves as the substrate recognition subunit in a cullin 4A-based ubiquitin ligase in Schizosaccharomyces pombe. Taken together, our results demonstrate that the CSA complex is able to ubiquitinate CSB, thereby inducing its degradation at late stages of the repair process.

We next explored the function of CSB degradation in the repair process. CSB is degraded at a specific stage of the TCR, corresponding to recovery of RNA synthesis (post-TCR RRS). Moreover, it has been previously shown that RNA synthesis recovery is sensitive to proteasome inhibitors (McKay et al. 2001), indicating that protein degradation is required for transcription to resume. In normal primary cells, as well as in immortalized fibroblasts, nascent mRNA synthesis was, indeed, sensitive to MG132 (Fig. 5A,B). Thus, inhibiting the proteasome blocked RNA transcription recovery and, at the same time, resulted in CSB persistence in the CSA complex (Fig. 2A). In order to test whether the effect of MG132 on post-TCR RRS could be directly linked to CSB degradation, we tested the effects of MG132 on the recovery of transcription in primary and SV40-transformed CS-B fibroblasts (CS1AN fibroblasts) (Fig. 5A,B). CS-B fibroblasts are derived from Cockayne syndrome patients and express a truncated (336-amino-acid N-terminal portion) CSB protein. Because of defective CSB, CS1AN fibroblasts exhibit poor post-TCR RRS. However, and in contrast to wild-type cells, residual transcription was insensitive to MG132 in CSB cells (Fig. 5A). This result indicates that the effect of MG132 in the wild-type cells is, at least in great part, due to inhibition of CSB degradation. Moreover, this result is corroborated by the previous demonstration that recovery of transcription in CS-A cells, but not in TCR-defective p53 ${ }^{-/-}$HCT116 cells, is insensitive to proteasome inhibitor, similar to CS-B cells (McKay et al. 2001). Finally, sensitivity to MG132 was restored by ectopic expression of wild-type CSB (Fig. 5A), further confirming the importance of CSB degradation for post-TCR RRS.

Taken together, our data indicate that CSB elimination by CSA is important for post-repair recovery. Interestingly, expression of mutant CSB is more detrimental than mere elimination of the allele (Horibata et al. 2004). A tempting hypothesis is that mutated CSB is not eliminated at the onset of recovery, thereby impairing the recovery process. To test this hypothesis, we monitored the levels of truncated CSB protein in UV-irradiated CS1AN fibroblasts (Fig. 5B), using an anti-CSB Ab that can detect both wild-type and truncated CSB (Horibata et al. 2004). In contrast to wild-type CSB, truncated CSB levels did not decrease during transcription recovery, and were insensitive to the treatment by proteasome inhibitors. (Fig. 5B). Thus the inability to degrade mutant CSB might affect transcription recovery in the patients' cells. The fact that truncated CSB is retained in the nucleus and colocalized with DNA in CS1AN cells (Horibata et al. 2004) raises the possibility that mutant CSB cannot be removed from the DNA template and likely becomes an obstacle for transcription recovery. These data support the relevance of CSB degradation for the Cockayne syndrome disease.

Taken together, our results demonstrate that CSA is a substrate receptor subunit of the SCF-like ubiquitin ligases that are connected to the cullin 4A scaffold
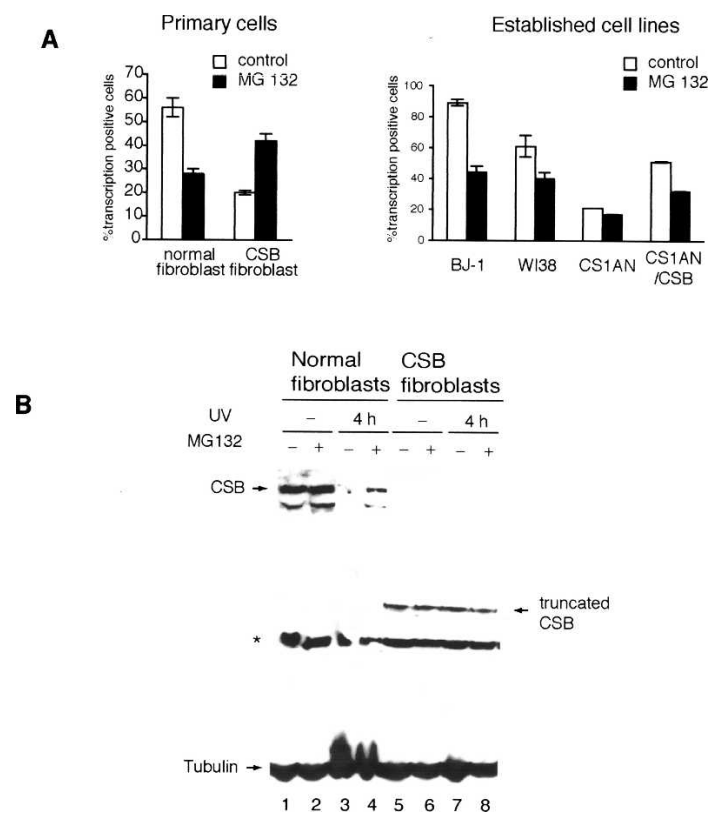

Figure 5. Proteasomal degradation of CSB is important for postTCR recovery of transcription. (A) Proteasome inhibition decreases the recovery of mRNA synthesis following UV irradiation. Nascent mRNA synthesis was assessed in the absence or presence of $12 \mu \mathrm{M}$ MG132 (black) $4 \mathrm{~h}$ following exposure to $20 \mathrm{~J} / \mathrm{m}^{2}$ of UV light in primary cells and $6 \mathrm{~h}$ following exposure to $10 \mathrm{~J} / \mathrm{m}^{2}$ of UV light in cell lines. $(B)$ Mutated CSB of CSB fibroblasts cannot be degraded by proteasome during transcription recovery. Amounts of wild-type and truncated CSB in the whole-cell lysates were measured on immunoblots with anti-CSB antibodies and normalized using $\alpha$-tubulin expression. The band labeled with an asterisk is nonspecific. 
through the DDB1 protein. We identified the DNA-dependent ATPase CSB, another complementation factor of Cockayne syndrome, as the first characterized substrate of the CSA ligase. Remarkably, CSB degradation starts only $3 \mathrm{~h}$ after UV-irradiation, following dissociation of the negative regulator COP9/CSN from the complex (Groisman et al. 2003), suggesting that the signalosome is involved in the signaling pathway that induces degradation. Our data support a model in which CSB has to be removed from the DNA template by CSA-dependent degradation in order for transcription to resume at a normal rate. This is in apparent contradiction with previous results unambiguously demonstrating an essential role for CSB in the transcription process. We propose that CSB acts at early stages of the process, either on repair itself or on transcription initiation following repair. Later, however, it becomes detrimental and has to be removed. This hypothesis is supported by the milder phenotype of CSB-null patients as compared with Cockayne syndrome caused by mutation in the protein (Horibata et al. 2004). Defects in CSB degradation/processing is reminiscent of other neurodegenerative disorders such as Parkinson's and Lou-Gehrig's diseases that are often associated with impaired ubiquitin-dependent protein degradation (Ciechanover and Brundin 2003; von Coelln et al. 2004). This novel mechanism for the regulation of post-TCR recovery can contribute to the efficiency of the TCR pathway. In summary, our study demonstrates a functional relationship between two complementation groups of the Cockayne syndrome but also provides the first evidence for the role of the ubiquitindependent degradation at the post-TCR recovery steps.

\section{Materials and methods}

Cells

eCSA:HeLa and eDDB2:HeLa S3 cells expressing Flag,HA-tagged CSA and DDB2, respectively, were maintained as described before (Groisman et al. 2003). SV40-immortalized CS-A fibroblasts CS3BE.S3.G1 stably transfected with a control vector pDR2 or pDR2-mycCSA, SV40-immortalized CS-B fibroblasts, CS1AN, and normal SV40-immortalized cell lines (VH1O) were provided by the E.C. Friedberg laboratory and were maintained as described (Bregman et al. 1996). For UV-irradiation experiments, HeLa cells and fibroblasts were grown on tissue culture dishes, washed with PBS, irradiated with UV at $25 \mathrm{~J} / \mathrm{m}^{2}$, and incubated in fresh media for the period indicated. Where indicated, proteasome inhibitor MG132 (Sigma) was added to cell media at $25 \mu \mathrm{M}$ concentration $1 \mathrm{~h}$ before UV irradiation and maintained until cell lysis. Cells were lysed in a buffer containing $0.5 \mathrm{M} \mathrm{KCl}, 20 \mathrm{mM}$ Tris- $\mathrm{HCl}$ ( $\mathrm{pH}$ 8.0), $0.2 \mathrm{mM}$ EDTA, $0.1 \%$ Tween, $10 \%$ glycerol, Complete TM protease inhibitor cocktail (Roche), and $25 \mu \mathrm{M}$ MG132 and were frozen immediately after lysis. Lysates, precleared by centrifugation at $2000 \times \mathrm{g}$ for $10 \mathrm{~min}$ at $4^{\circ} \mathrm{C}$ were used for direct immunoblotting or immunoprecipitation with indicated antibodies. The CSA immunoprecipitated complex was purified from nuclear extracts prepared from HeLa cells expressing the CSA protein fused with C-terminal Flag- and HA-epitope tags (e-CSA) by immunoprecipitation on anti-Flag antibody-conjugated agarose. The bound polypeptides were eluted with the Flag peptide (Groisman et al. 2003). For density gradient sedimentation, $0.5 \mathrm{~mL}$ of the Flag peptide-eluted material was loaded onto a $4-\mathrm{mL}$ glycerol gradient $(10 \%-40 \%)$ and spun at $368,000 \mathrm{~g}$ in a Beckman SW55Ti rotor for $2 \mathrm{~h} ; 200-\mu \mathrm{L}$ fractions were collected from the top of the gradient. For RRS assays, primary and stable cell lines were used. Normal human primary fibroblasts (AS198) were isolated by explant culture of a 6 -mo-old boy foreskin sample. CS-B Cockayne's primary fibroblasts were isolated by explant culture of a nonphoto-exposed skin biopsy taken from the buttock of a 2-yr-old boy. Cells were cultured in DMEM medium containing 10,000 IU of penicillinstreptomycin, $1 \mathrm{mM}$ sodium pyruvate, $0.1 \mathrm{mM}$ nonessential amino acids, and $2 \mathrm{mM} \mathrm{L}$-glutamine. All analyses were carried out using cells at passages $5-8$.
Normal human BJ1 fibroblasts immortalized by hTERT, SV40-transformed CS-B fibroblasts, and CS-B fibroblasts stably transfected with wild-type CSB were grown as described previously (Horibata et al. 2004).

\section{Recovery of RNA synthesis after UV irradiation}

Primary fibroblasts were grown for $24 \mathrm{~h}$ on glass coverslips at a density of 10,000 cells $/ \mathrm{cm}^{2}$ in Ham's-F10 containing $15 \%$ fetal bovine serum and $10,000 \mathrm{IU}$ of penicillin-streptomycin. Cells were then incubated for $24 \mathrm{~h}$ in Ham's-F10 medium containing 3\% dialyzed serum and antibiotics. On day 3, $12.5 \mu$ M MG132 in DMSO or DMSO alone was added to the cells at the time of mock irradiation or irradiation. UVC irradiation was carried out using a UVC $(254 \mathrm{~nm})$ tube at doses of $20 \mathrm{~J} / \mathrm{m}^{2}$. After indicated times $(4 \mathrm{~h})$, RNA synthesis was labeled for $1 \mathrm{~h}$ in the presence of 10 $\mu \mathrm{Ci} / \mathrm{mL}^{3} \mathrm{H}$-Uridine (Amersham). Cells were then washed three times in PBS, and fixed in methanol for $10 \mathrm{~min}$. Two TCA $(5 \%)$ precipitations were then carried out before ethanol dehydration and autoradiography of mounted coverslips using NTB1 emulsion (Kodak). Slides were developed for 24 h, and then revealed and fixed in Kodak D19 and Kodak 3000 solutions, respectively. Cell nuclei were then counterstained using Meyer's hematoxylin solution. After mounting, autoradiographic grains over nuclei were observed under a $\times 100$ immersional microscope and counted using the image analysis Alcatel TINT device equipped with the Autoradio 3.09 software. For each experimental condition, 125-200 intact nuclei were counted.

BJ1- and SV40-transformed normal and CS-B cell lines were treated with $12 \mu \mathrm{M}$ MG132 in DMSO, exposed to UV light $\left(10 \mathrm{~J} / \mathrm{m}^{2}\right)$, and labeled with ${ }^{3} \mathrm{H}$-uridine in the same way as primary cells $6 \mathrm{~h}$ after irradiation. Incorporation of ${ }^{3} \mathrm{H}$-uridine in nascent RNA synthesis was measured as described in Horibata et al. (2004).

Reconstitution of the DDB2 and CSA complexes containing ubiquitin E3 ligases from recombinant proteins

Recombinant GST-tagged CUL4A (a gift of Dr. Hui Zhang) was coexpressed with recombinant Roc1 (gift of Dr. Nikola Pavletich) in Sf9 cells via the Bac-to-Bac baculovirus expression system (Invitrogen). The GSTCUL4A/Roc1 heterodimer was purified from Sf9 extracts by glutathioneSepharose chromatography. The GST moiety was cleaved using biotinylated thrombin, and the thrombin was removed using streptavidinagarose, using the Novagen Thrombin Cleavage/Capture Kit. Recombinant HA-tagged DDB1 was coexpressed with recombinant Flag-tagged DDB2 or CSA in the same system. In order to establish a stoichiometric ratio for all subunits of the DDB2 and CSA E3 ligases, HA-tagged DDB1/ Flag-tagged DDB2 or Flag-CSA heterodimers were bound to the antiHA antibody-conjugated agarose, eluted with $250 \mu \mathrm{g} / \mathrm{mL}$ HA-peptide (Covance), bound to the anti-Flag agarose, and then incubated with the excess of purified CUL4A/Rocl heterodimer. After removal of the unbound CUL4A/Roc1 heterodimer, stoichiometric complexes were then removed from the agarose by elution with $200 \mu \mathrm{g} / \mathrm{mL}$ Flag-peptide (Sigma).

In vitro ubiquitin ligase assay

To determine whether CSB is ubiquitinated by CSA or DDB2 E3 ligases in vitro, $0.1 \mu \mathrm{g}$ of purified recombinant CSB (gift of Drs. Christopher P. Selby and Aziz Sancar) (Selby and Sancar 1997b) was incubated with 0.04 $\mu \mathrm{g}(0.08 \mu \mathrm{g})$ of the in vitro reconstituted CSA or DDB2 E3 ligases, $0.1 \mu \mathrm{g}$ of Ubal E1, $0.03 \mu \mathrm{g}$ of UbcH5b E2, and $5 \mu \mathrm{g}$ of ubiquitin in $15 \mu \mathrm{L}$ of assay buffer containing $50 \mathrm{mM}$ Tris- $\mathrm{HCl}(\mathrm{pH} 8.0), 5 \mathrm{mM} \mathrm{MgCl}_{2}, 0.2 \mathrm{mM} \mathrm{CaCl}_{2}$, $1 \mathrm{mM} \mathrm{DTT}$, and $4 \mathrm{mM}$ ATP. After $60 \mathrm{~min}$ of incubation at $30^{\circ} \mathrm{C}$, reaction mixtures were separated by SDS-PAGE, and modifications of CSB and CUL4A were analyzed by immunoblotting with anti-CSB (a kind gift of Drs. Christopher P. Selby and Aziz Sancar) and anti-CUL4A (a kind gift of Dr. Pradip Raychaudhuri) antibodies.

\section{Acknowledgments}

We thank D. Finley for critical review of the manuscript; J.-M. Egly, R. Drapkin, and L. Myers for discussion; A. Sarasin for CS-B Cockayne's primary fibroblasts; A. Herlitz and P. Fischhaber for technical assistance, and D. Goff for editing text. These studies were supported by an HFSP grant to Y.N. A.F.K. was supported by a Special fellowship from the Leukemia and Lymphoma Society. 


\section{References}

Beerens, N., Hoeijmakers, J.H., Kanaar, R., Vermeulen, W., and Wyman, C. 2005. The CSB protein actively wraps DNA. J. Biol. Chem. 280 4722-4729.

Bootsma, D., Kreamer, K.H., Cleaver, J.E., and Hoeijmakers, J.H. 1998. Nucleotide excision repair syndromes: Xeroderma pigmentosum, Cockayne syndrome, and trichothiodystrophy. In The gentetic basis of human cancer (eds. B. Vogelsteain and K.W. Kinzler), pp. 254-257. McGraw-Hill, New York.

Bradsher, J., Auriol, J., Proietti de Santis, L., Iben, S., Vonesch, J.L., Grummt, I., and Egly, J.M. 2002. CSB is a component of RNA Pol I transcription. Mol. Cell 10: 819-829.

Bregman, D.B., Halaban, R., van Gool, A.J., Henning, K.A., Friedberg E.C., and Warren, S.L. 1996. UV-induced ubiquitination of RNA polymerase II: A novel modification deficient in Cockayne syndrome cells. Proc. Natl. Acad. Sci. 93: 11586-11590.

Ciechanover, A. and Brundin, P. 2003. The ubiquitin proteasome system in neurodegenerative diseases: Sometimes the chicken, sometimes the egg. Neuron 40: 427-446.

Citterio, E., Rademakers, S., van der Horst, G.T., van Gool, A.J., Hoeijmakers, J.H., and Vermeulen, W. 1998. Biochemical and biological characterization of wild-type and ATPase-deficient Cockayne syndrome B repair protein. J. Biol. Chem. 273: 11844-11851.

Citterio, E., Van Den Boom, V., Schnitzler, G., Kanaar, R., Bonte, E. Kingston, R.E., Hoeijmakers, J.H., and Vermeulen, W. 2000. ATPdependent chromatin remodeling by the Cockayne syndrome B DNA repair-transcription-coupling factor. Mol. Cell. Biol. 20: 7643-7653.

Friedberg, E.C., Walker, G.C., and Siede, W. 1995. DNA repair and mutagenesis. American Society for Microbiology, Washington, DC.

Groisman, R., Polanowska, J., Kuraoka, I., Sawada, J., Saijo, M., Drapkin, R., Kisselev, A.F., Tanaka, K., and Nakatani, Y. 2003. The ubiquitin ligase activity in the DDB2 and CSA complexes is differentially regulated by the COP9 signalosome in response to DNA damage. Cell 113: $357-367$.

Henning, K.A., Li, L., Lyer, N., McDaniel, L., Reagan, M.S., Legersky, R., Schults, R.A., Stefanini, M., Lehmann, A.R., Mayne, L.V., et al. 1995. The Cockayne syndrome group A gene encodes a WD repeat protein that interacts with CSB protein and a subunit of RNA polymerase II TFIIH. Cell 82: 555-564.

Horibata, K., Iwamoto, Y., Kuraoka, I., Jaspers, N.G., Kurimasa, A., Oshimura, M., Ichihashi, M., and Tanaka, K. 2004. Complete absence of Cockayne syndrome group B gene product gives rise to UV-sensitive syndrome but not Cockayne syndrome. Proc. Nat1. Acad. Sci. 101: 15410-15415

Kamiuchi, S., Saijo, M., Citterio, E., de Jager, M., Hoeijmakers, J.H., and Tanaka, K. 2002. Translocation of Cockayne syndrome group A protein to the nuclear matrix: Possible relevance to transcriptioncoupled DNA repair. Proc. Natl. Acad. Sci. 99: 201-206.

Licht, C.L., Stevnsner, T., and Bohr, V.A. 2003. Cockayne syndrome group B cellular and biochemical functions. Am. J. Hum. Genet. 73: 1217-1239

Liu, C., Poitelea, M., Watson, A., Yoshida, S.H., Shimoda, C., Holmberg, C., Nielsen, O., and Carr, A.M. 2005. Transactivation of Schizosaccharomyces pombe cdt2+ stimulates a Pcu4-Ddb1-CSN ubiquitin ligase. EMBO J. 24: 3940-3951.

McKay, B.C., Chen, F., Clarke, S.T., Wiggin, H.E., Harley, L.M., and Ljungman, M. 2001. UV light-induced degradation of RNA polymerase II is dependent on the Cockayne's syndrome A and B proteins but not p53 or MLH1. Mutat. Res. 485: 93-105.

Petroski, M.D. and Deshaies, R.J. 2005. Function and regulation of Cullin-RING ubiquitin ligases. Nat. Rev. Mol. Cell Biol. 6: 9-20.

Rockx, D., Mason, R., van Hoffen, A., Barton, M., Citterio, E., Bregman, D.B., van Zeeland, A.A., Vrieling, H., and Mullenders, L.H. 2000 UV-induced inhibition of transcription involves repression of transcription initiation and phosphorylation of RNA polymerase II. Proc. Nat1. Acad. Sci. 97: 10503-10508.

Selby, C.P. and Sancar, A. 1997a. Cockayne syndrome group B protein enhances elongation by RNA polymerase II. Proc. Natl. Acad. Sci. 94: 11205-11209.

- 1997b. Human transcription-repair coupling factor CSB/ERCC6 is a DNA-stimulated ATPase but is not a helicase and does not disrupt the ternary transcription complex of stalled RNA polymerase II.
J. Biol. Chem. 272: 1885-1890.

Svejstrup, J.Q. 2002. Mechanisms of transcription-coupled DNA repair. Nat. Rev. Mol. Cell Biol. 3: 21-29.

. 2003. Rescue of arrested RNA polymerase II complexes. I. Cell Sci. 116: 447-451.

Tantin, D., Kansal, A., and Carey, M. 1997. Recruitment of the putative transcription-repair coupling factor CSB/ERCC6 to RNA polymerase II elongation complexes. Mol. Cell. Biol. 17: 6803-6814.

Troelstra, C., van Gool, A., de Wit, J., Vermeulen, W., Bootsma, D., and Hoeijmakers, J.H. 1992. ERCC6, a member of a subfamily of putative helicases, is involved in Cockayne's syndrome and preferential repair of active genes. Cell 71: 939-953.

van der Horst, G.T., van Steeg, H., Berg, R.J., van Gool, A.J., de Wit, J., Weeda, G., Morreau, H., Beems, R.B., van Kreijl, C.F., de Gruijl, F.R. et al. 1997. Defective transcription-coupled repair in Cockayne syndrome B mice is associated with skin cancer predisposition. Cell 89: 425-435.

van Gool, A.J., Citterio, E., Rademakers, S., van Os, R., Vermeulen, W. Constantinou, A., Egly, J.M., Bootsma, D., and Hoeijmakers, J.H. 1997. The Cockayne syndrome B protein, involved in transcriptioncoupled DNA repair, resides in an RNA polymerase II-containing complex. EMBO T. 16: 5955-5965.

von Coelln, R., Dawson, V.L., and Dawson, T.M. 2004. Parkin-associated Parkinson's disease. Cell Tissue Res. 318: 175-184.

Wolf, D.A., Zhou, C., and Wee, S. 2003. The COP9 signalosome: An assembly and maintenance platform for cullin ubiquitin ligases? Nat. Cell Biol. 5: 1029-1033.

Yu, A., Fan, H.Y., Liao, D., Bailey, A.D., and Weiner, A.M. 2000. Activation of p53 or loss of the Cockayne syndrome group B repair protein causes metaphase fragility of human U1, U2, and 5S genes. Mol. Cell 5: 801-810. 


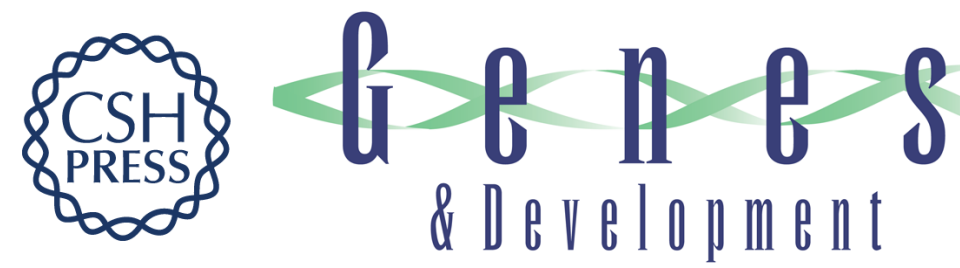

\section{CSA-dependent degradation of CSB by the ubiquitin-proteasome pathway establishes a link between complementation factors of the Cockayne syndrome}

Regina Groisman, Isao Kuraoka, Odile Chevallier, et al.

Genes Dev. 2006, 20:

Access the most recent version at doi:10.1101/gad.378206

References This article cites 26 articles, 12 of which can be accessed free at:

http://genesdev.cshlp.org/content/20/11/1429.full.html\#ref-list-1

License

Email Alerting Receive free email alerts when new articles cite this article - sign up in the box at the top Service right corner of the article or click here.

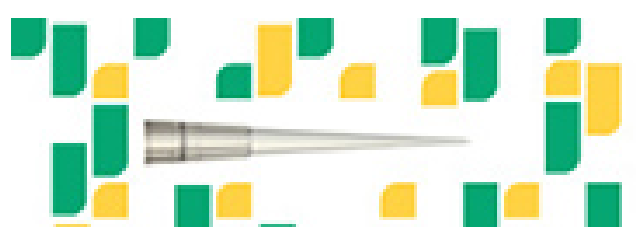

Focused on your science. 